\title{
A SEARCH FOR PLANETARY ECLIPSES OF WHITE DWARFS IN THE Pan-STARRS1 MEDIUM-DEEP FIELDS
}

\author{
B. J. Fulton, J. L. Tonry, H. Flewelling, W. S. Burgett, K. C. Chambers, K. W. Hodapp, \\ M. E. Huber, N. Kaiser, R. J. WainscoAT, AND C. WATERS \\ Institute for Astronomy, University of Hawaii at Manoa, Honolulu, HI 96822, USA \\ Received 2014 June 27; accepted 2014 September 21; published 2014 November 13
}

\begin{abstract}
We present a search for eclipses of $\sim 1700$ white dwarfs (WDs) in the Pan-STARRS1 medium-deep fields. Candidate eclipse events are selected by identifying low outliers in over 4.3 million light curve measurements. We find no short-duration eclipses consistent with being caused by a planetary size companion. This large data set enables us to place strong constraints on the close-in planet occurrence rates around WDs for planets as small as $2 R_{\oplus}$. Our results indicate that gas giant planets orbiting just outside the Roche limit are rare, occurring around less than $0.5 \%$ of WDs. Habitable-zone super-Earths and hot super-Earths are less abundant than similar classes of planets around main-sequence stars. These constraints provide important insight into the ultimate fate of the large population of exoplanets orbiting main-sequence stars.
\end{abstract}

Key words: planets and satellites: detection - white dwarfs

Online-only material: color figures

\section{INTRODUCTION}

Searches for planets outside our solar system have focused primarily on hydrogen-burning main-sequence stars similar to our Sun (e.g., Bakos et al. 2004; Howard et al. 2010a; Borucki et al. 2010). As we discovered that planets are nearly ubiquitous in our solar neighborhood (Howard et al. 2010b) and in the Kepler field (Petigura et al. 2013), searches around M-dwarfs gained popularity (e.g., Nutzman \& Charbonneau 2008). Studies of M-dwarfs enjoy a boost in sensitivity to small planets because transits block a larger fraction of the stellar disk and induce a larger amplitude reflex motion of the star around the barycenter due to their low mass. Some studies have also searched for and explored the planet occurrence rates as a function of stellar mass from M-dwarfs to intermediate-mass subgiants (Johnson et al. 2007). Microlensing campaigns survey stars of many types and are sensitive to planets around all massive hosts regardless of their stage in stellar evolution (Gaudi 2012), but follow up characterization of these planets is impossible. However, there have been few dedicated searches for planets around white dwarfs (WDs).

Many studies, including Mullally (2007), Farihi et al. (2008), and Kilic et al. (2009), searched for infrared-excess indicative of planetary companions to WDs. They detected several brown dwarf companions (Zuckerman \& Becklin 1992; Farihi et al. 2005; Steele et al. 2009) but no planetary-mass objects. Mullally (2007) also searched for companions using the pulsations of WDs to look for periodic deviations in the pulse arrival times caused by an orbiting companion. They find evidence of a $2.4 M_{J}$ companion in a $4.6 \mathrm{yr}$ orbit. Hogan et al. (2009) and Debes et al. (2005) conducted high-contrast imaging surveys of nearby WDs to search for low-mass companions at large separations. Burleigh et al. (2006) found a brown dwarf in the near-IR spectrum of WD 0137-349 with an orbital period of only two hours. This object may have survived the common-envelope phase or migrated from larger orbital distances after the formation of the WD. Faedi et al. (2011) conduct a transit search for a sample of 174 WDs using SuperWASP data (Pollacco et al. 2006) and find no eclipsing companions, but can place only weak constraints on the planet occurrence rates due to their small sample size ( $<10 \%$ for Jupiter-size planets). Drake et al. (2010) search for eclipses of $\sim 12,000$ color-selected WDs using Catalina Sky Survey photometry and Sloan Digital Sky Survey spectroscopy. They find 20 eclipsing systems, 3 of which have radii consistent with substellar objects and no detectable flux in the spectra.

WDs have radii only $\sim 1 \%$ of the Sun, or about the same size as the Earth. This implies that an Earth-sized object transiting the WD with an impact parameter of 1.0 would cause a complete occultation. Although these occultations are short-duration, they can be easily detected from small ground-based telescopes with short exposure times and relatively low photometric precision (Drake et al. 2010). In addition, the most common WDs are old and cool with surface temperatures of $\sim 5000 \mathrm{~K}$. Their small radii and low surface temperatures imply that their luminosity is low, with typical values of $\sim 10^{-4} L_{\odot}$, and the habitable zone (HZ) is close-in ( $a \sim 0.01 \mathrm{AU}$; Agol 2011), giving rise to significant transit probabilities. This makes Earth-size planets orbiting in the HZs of old, cool WDs relatively easy to detect via the transit method.

Most main-sequence stars, including our Sun, will eventually end their lives slowly cooling as WDs. Since approximately $50 \%$ of main-sequence stars host at least one planet (Mayor et al. 2011), it is interesting to consider their fate as the star evolves into a WD. It is unlikely that any planets inside $\sim 1 \mathrm{AU}$ would survive being engulfed by their host stars as they expand onto the red giant branch, but it is unclear what becomes of the planetary debris. Since WDs quietly cool for the age of the universe, it is conceivable that new planets could form out of the debris of a previous generation of planets. The migration of planets from outside of $1 \mathrm{AU}$ is also plausible, but is likely a rare occurence (Mustill et al. 2014). Several studies have identified pollution by heavy elements on the surfaces of WDs (Zuckerman et al. 2010) and IR excess indicative of a debris disk (Debes et al. 2011). Extensive work has been done to identify the chemical composition of this pollution. Silicates and glasses were detected in the atmospheres of six WDs by Jura et al. (2009) and interpreted as signs of the accretion 


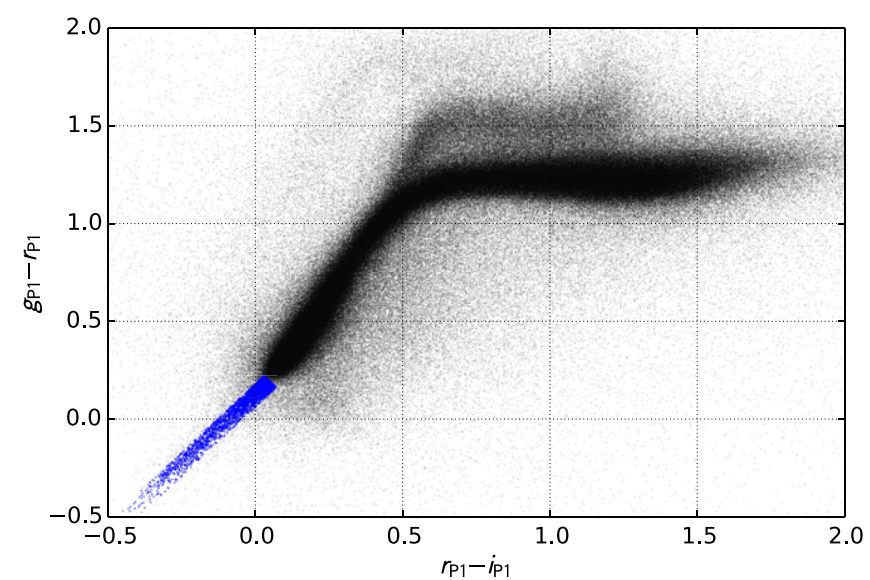

Figure 1. Color-selected WDs (blue points) are identified by the narrow tail of extremely blue stars in the $\left(g_{\mathrm{P} 1}-r_{\mathrm{P} 1}\right)$ vs. $\left(r_{\mathrm{P} 1}-i_{\mathrm{P} 1}\right)$ color plane. The small black points are all detections from the deep stacks not selected for either the control or WD samples.

(A color version of this figure is available in the online journal.)

of asteroid-like bodies onto the WD. A detailed study by $\mathrm{Xu}$ et al. (2014) using data from the Keck telescope and Hubble Space Telescope showed strong evidence that the composition of metals in the atmospheres of WDs G29-38 and GD 133 closely mirror the composition of the bulk Earth, furthering the idea that close-in terrestrial planets orbit and eventually accrete onto WDs.

We present a systematic search for eclipses of WDs by planetary-size objects in the Pan-STARRS1 medium-deep fields (Tonry et al. 2012). We use a combination of astrometric and photometric selection techniques to identify 3179 WDs with a range of ages and temperatures. Each WD was observed on 1000-3000 epochs during the past five years for a total of 4.3 million measurements. Although we do not detect any substellar companions, this large number of observations allows us to place tight constraints on the occurrence rates of planets orbiting WDs.

\section{METHODS}

\subsection{WD Sample}

We analyze a total of 3179 WD candidates spread across the 10 medium-deep fields spanning $70 \mathrm{deg}^{2}$ on the sky. Each field is observed on 1000-3000 epochs with four to eight consecutive $240 \mathrm{~s}$ exposures per night. Our sample of WDs is segregated into two categories. We identify 661 targets using their proper motions as described in Tonry et al. (2012; astrometric sample hereafter). These objects have a high probability of being bona fide WDs and a very low contamination rate.

The remaining 2518 WDs were selected based on their photometric colors (color-selected sample hereafter). We use the following criteria to select the locus of hot, blue stars from the $\left(g_{\mathrm{P} 1}-r_{\mathrm{P} 1}\right)$ versus $\left(r_{\mathrm{P} 1}-i_{\mathrm{P} 1}\right)$ color plane shown in Figure 1: $\left(g_{\mathrm{P} 1}-r_{\mathrm{P} 1}\right)<0.18+1.4\left(r_{\mathrm{P} 1}-i_{\mathrm{P} 1}\right),\left(g_{\mathrm{P} 1}-r_{\mathrm{P} 1}\right)>$ $0.06+1.4\left(r_{\mathrm{P} 1}-i_{\mathrm{P} 1}\right),\left(g_{\mathrm{P} 1}-r_{\mathrm{P} 1}\right)<0.25-1.25\left(r_{\mathrm{P} 1}-i_{\mathrm{P} 1}\right)$, and $i_{\mathrm{P} 1}<22$. This sample is restricted to hot WDs due to the requirement of blue colors and is likely contaminated by other hot stars.

To quantify the contamination rate of the color-selected sample, we created a Besancon galactic simulation of the

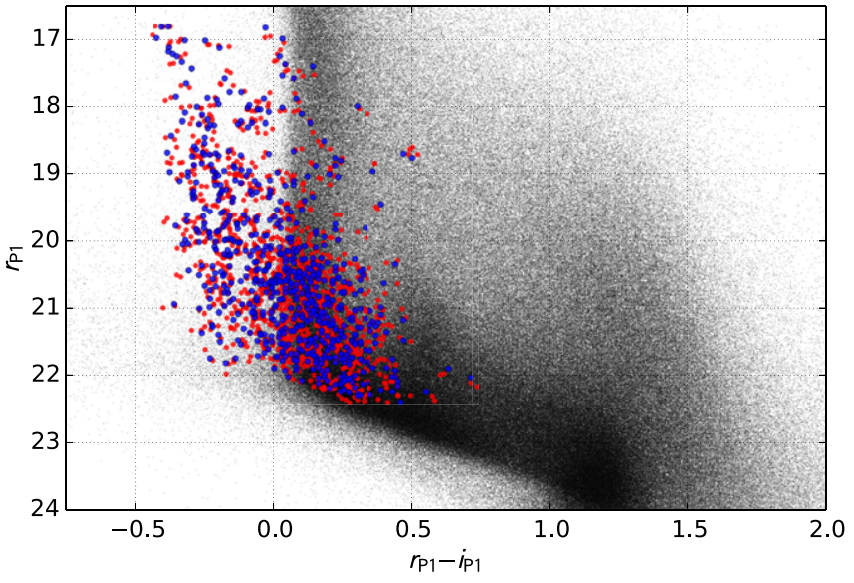

Figure 2. Astrometrically selected WDs (blue) and control sample stars (red). The small black points are all detections from the deep stacks that were not selected for either the control or WD samples.

(A color version of this figure is available in the online journal.)

medium-deep fields (Robin et al. 2003). When we make the same color cuts we find that $42 \%$ of the stars are bona fide WDs according to the model. The stars that are within this locus but not WDs are mostly distant A- and B-type subdwarfs in the halo of the galaxy. Closer F-type subdwarfs would also fall into the locus, but are mostly far too bright to be included in our sample. We also find that the contamination rate is highly dependent on apparent magnitude with the fainter stars being much more likely to be WDs. We assume a $58 \%$ contamination rate for our color-selected sample for all further analysis. This reduces our total number of WDs to 1718 .

\subsection{Control Sample}

Our control sample consists of stars with similar magnitudes and colors to the astrometrically selected WDs but with undetectable proper motions. These should be relatively hot stars with radii much larger than WDs around which we would not expect to see the very short-duration eclipses indicative of a planet occulting a WD. We can compare the number of potential eclipses found in the WD sample to the number that we find in the control sample to better understand the frequency of eclipse-like events caused by non-astrophysical effects.

We select the control sample by binning the astrometric sample of WDs in two-dimensional color bins of $r_{\mathrm{P} 1}$ versus $\left(r_{\mathrm{P} 1}-i_{\mathrm{P} 1}\right)$. For each bin that contains at least one WD, we select two times the number of WDs in that bin from a sample of all stellar detections derived from deep stacks of the medium-deep fields, excluding stars that are already part of the WD samples. Figure 2 shows our control sample and astrometric WD sample in the $r_{\mathrm{P} 1}$ versus $\left(r_{\mathrm{P} 1}-i_{\mathrm{P} 1}\right)$ color plane. If fewer than three field stars are available in a particular bin, then we select all of the available stars. This produces a total of 1296 stars for the control sample which is later trimmed down to 1288 by removing RR-Lyrae, Delta-Scuti, and other variable stars (see Section 2.4).

\subsection{Light Curves}

Light curves are extracted for each WD and control sample star by directly analyzing the first-level Pan-STARRS1 
photometry product (SMF files). These SMF files consist of the raw photometry extracted from the calibrated images before a zero-point or precise world coordinate system (WCS) is established. Each camera exposure corresponds to a single SMF file. For each SMF file, we first find the WCS solution in order to associate pixel locations with sky positions. We then associate the per-image detections with detections in deep stacks for each field and extract the point-spread-function (PSF)-fitted photometry to obtain raw instrumental magnitudes. We fit for the photometric zero-point using the technique described in (Schlafly et al. 2012). The instrumental magnitudes for all detections within 5 arcmin of the target are also extracted and recorded along with the target instrumental magnitudes. All of the epochs for which a target could not be matched to a detection in the SMF file are carefully recorded and the neighboring star photometry is still extracted if available. This ensures that we are sensitive to large decreases in flux that may cause the target to fall below the detection threshold in a particular image, and in some cases we can use the photometric statistics of the neighboring stars to explain the non-detection. We also record the pixel locations relative to the entire CCD array and particular chip for each epoch.

\subsection{Eclipse Detection}

Since eclipses are rare and extremely short duration, traditional periodic search algorithms, such as the box-least-squares (BLS) periodogram (Kovács et al. 2002), fail to recover such signals. BLS excels at detecting signals in the regime of many transits with low single-event signal-to-noise ratio $(\mathrm{S} / \mathrm{N})$ but planetary eclipses of our target stars would produce very infrequent, but very deep, high $\mathrm{S} / \mathrm{N}$ eclipses. Instead, we employ an extremely simple eclipse detection technique. We look for low outliers in the light curves (dropouts) that are caused either by a complete non-detection or that show a deficit of flux relative to the median flux level $(\Delta F)$ that is greater than five times the measurement uncertainty $\left(\Delta F / \sigma_{\mathrm{lc}} \geqslant 5\right)$. Figure 3 shows the distribution of $\Delta F$ and $\Delta F / \sigma_{\mathrm{lc}}$ for all of the light curves.

The raw light curves are heavily contaminated with nondetections and large flux drops that could be indicative of an eclipse event or a variety of non-astrophysical scenarios. For every dropout, we first check that the star did not fall off of, or too near, the edge of a chip. We initially noted that the dropout events were concentrated around the edges of the chips. This is likely caused by the PSF fit failing due to a strong gradient in the background region near the edges of the chips. This effect is worse at the corners of the chips near the readout electronics. For these reasons, we remove all light curve measurements that fall within 10 pixels $\left(22^{\prime \prime} .5\right)$ of an edge or within 100 pixels $\left(25^{\prime \prime}\right)$ of a corner. We consider this filter unbiased with respect to eclipses because there is no reason to expect that real eclipses would preferentially occur when the stars fall near the edge of a chip. Measurements with reported positions that fall between chip gaps or off the array are also excluded at this stage. All non-detections are removed with these chip location-based filters.

If the photometry of the neighboring stars also show a large decrease in flux at the same time of the target dropout, then clouds or poor seeing are likely to blame. We exclude all measurements for which the median magnitude of the neighboring stars drops by more than 0.5 magnitudes or the
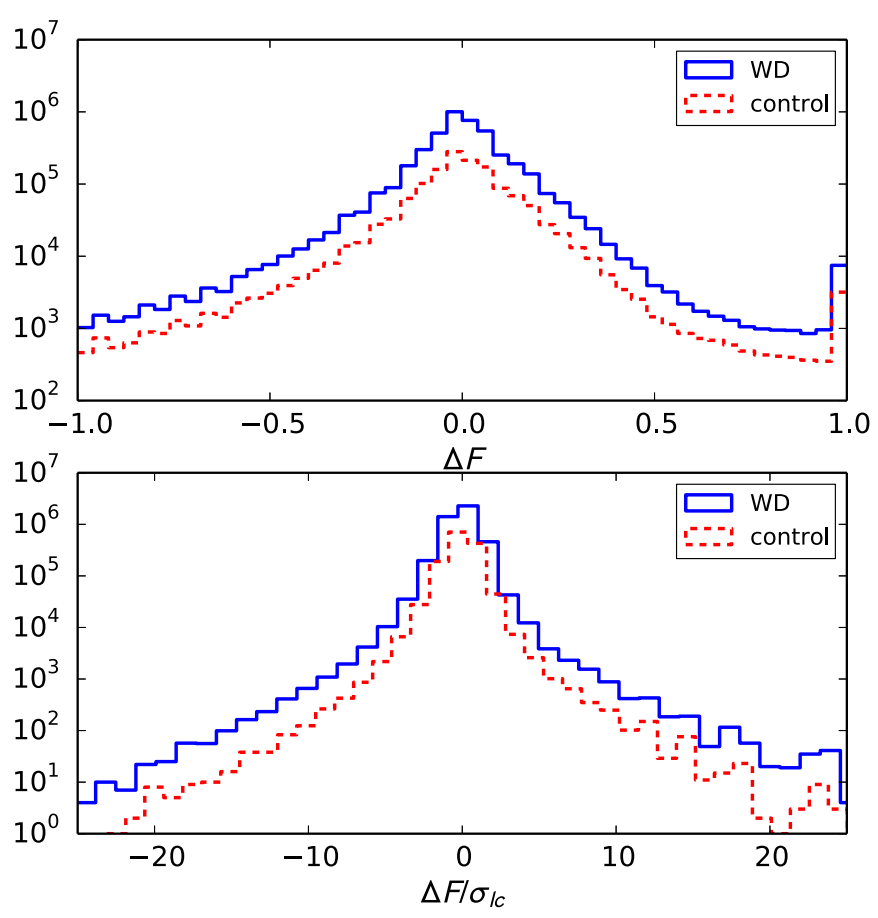

Figure 3. Top: distribution of relative flux measurements for all WD and control sample stars. The solid blue line is the distribution for the WDs and the dashed red line is for the control sample stars. Bottom: distribution of relative flux measurements divided by the measurement uncertainties corrected by adding in quadrature the reported measurement uncertainties with the standard deviation of the light curve (by filter). Measurements with $\Delta F / \sigma_{\mathrm{lc}} \geqslant 5$ are considered eclipse candidates. As in the top panel, the solid blue line is the distribution for the WDs and the dashed red line comes from the control sample stars.

(A color version of this figure is available in the online journal.)

standard deviation of the neighbor magnitudes is greater than one. We also de-correlate the target relative flux measurements against the median $\Delta F$ of the neighboring stars to reduce the effect of spatially dependent extinction.

Now that we have removed most of the egregious outliers from the light curve, we redefine the measurement errors. We sum in quadrature the reported measurement uncertainties with the median absolute deviation of the full light curve in each filter. This process always inflates the errors relative to the original measurement uncertainties and effectively removes many remaining candidate dropouts by decreasing the value of $\Delta F / \sigma_{\mathrm{lc}}$.

At this stage, we use the VARTOOLS package to create BLS and analysis-of-variance periodograms (Hartman et al. 2008; Kovács et al. 2002; Schwarzenberg-Czerny 1989; Devor 2005) for all WD and control sample stars. We visually inspect these periodograms and the light curves phase-folded to the ephemeris that corresponds to the highest peak in each periodogram. Obvious periodic variable stars are removed from further analysis. Thirty-three RR-Lyrae and Delta-Scuti stars, one dwarf nova (IY Uma), and three variables of unknown type are identified and removed at this stage.

For the remaining dropouts, we check their CCD locations against the regions of the array that are consistently masked by the Pan-STARRS1 Image Processing Pipeline (IPP). After applying all of the photometry-based tests, we are left with 11,570 potential dropout events and of a total of 4.3 million detections. 2570 of the dropout candidates are from the control sample and 
Table 1

Detection Statistics For All WDs

\begin{tabular}{lrrrrr}
\hline \hline Filter & \multicolumn{2}{c}{ WD } & & \multicolumn{2}{c}{ Control } \\
\cline { 2 - 3 } \cline { 5 - 6 } & $N_{\text {detections }}$ & $N_{\text {dropouts }}$ & & $N_{\text {detections }}$ & $N_{\text {dropouts }}$ \\
\hline No filters & 5650109 & 6963603 & & 1814296 & 3106873 \\
CCD location-based filters & 4757706 & 1771860 & & 1523212 & 622154 \\
Neighboring star filter & 4509855 & 1651266 & & 1439106 & 577904 \\
Re-calculate measurement errors & 4349232 & 15120 & & 1363979 & 3983 \\
Remove masked CCD regions & 4343011 & 9000 & & 1362535 & 2570 \\
\hline
\end{tabular}

the remaining 9000 are from the merged WD samples. This photometric filtering process for a single representative case is illustrated in Figure 4, and the total number of detections and non-detections removed at each stage in the filtering process are listed in Table 1.

We download the corresponding postage stamp images for any dropouts that make it through all of these light-curvebased tests for additional screening. In addition to the postage stamp corresponding to the dropout, we also download a deep stack around the target and the image that corresponds to the light curve measurement that is closest to the median value for that filter. We apply a few more automated filters before visually inspecting the remaining candidates. The images are automatically inspected for masking or CCD defects around the target that produce not-a-number (nan) values, very poor seeing, or clouds, as indicated by a low zero-point magnitude. We also perform aperture photometry on the three images and correct to an absolute apparent magnitude using the zero-point magnitude provided in the image headers. Our photometry acts as a check that the magnitude value reported by the IPP is in rough agreement with simple aperture photometry.

As a final step, we use the HOTPANTS implementation of the ISIS image subtraction software (Alard 2000) to produce a difference image using the deep stack as a template. We convolve the template to match the PSF and zero point of the dropout candidate image and subtract the convolved template from the candidate postage stamp. This difference image was used to aid the visual inspection of the 133 dropout events that could not be explained by any of the photometry or image-based filters. Figure 5 shows an example dropout candidate image and the image-differencing processes used for visual inspection. We find no eclipse with a duration compatible with an eclipse by a substellar object in any WD or control sample light curve.

\section{ANALYSIS}

\subsection{Theoretical Eclipse Probabilities}

In order to assess the likelihood that an occultation would have occurred during our observing window, we calculate the probability of eclipse as a function of the eclipse depth and then apply the noise properties and eclipse detection techniques that we used in our search. This tells us the number of eclipses we should have been able to detect as a function of the planet radius, orbital semi-major axis, and the occurrence rate of planets around WDs $(\eta)$.

The flux when a dark sphere eclipses a uniformly illuminated sphere is given by Equation (1) (Mandel \& Agol 2002), where $\kappa_{1}=\cos ^{-1}\left[\left(1-p^{2}+b^{2}\right) / 2 b\right], \kappa_{0}=\cos ^{-1}\left[\left(p^{2}+b^{2}-1\right) / 2 p b\right]$, and $p \equiv R_{p} / R_{\mathrm{WD}}$ is the planet-to-WD-radius ratio.

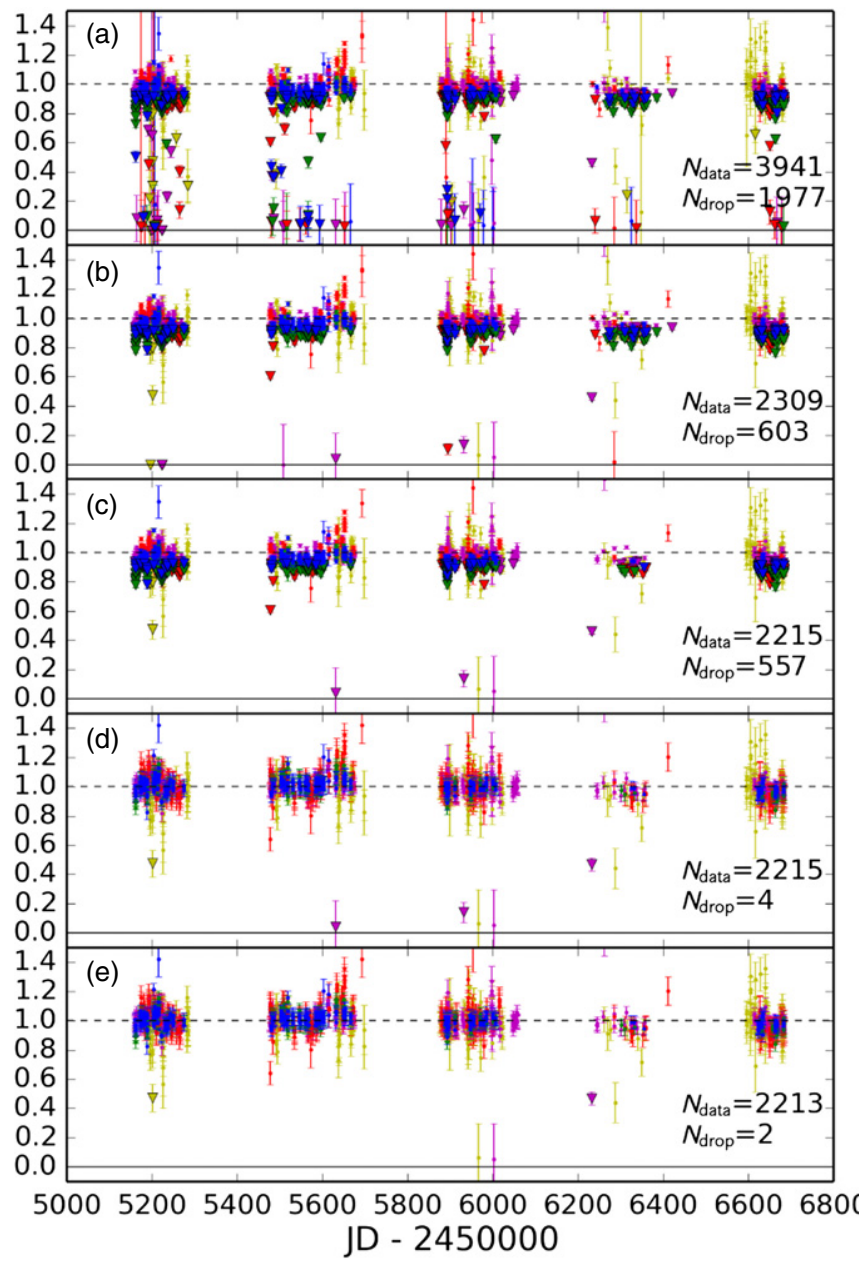

Figure 4. Illustration of the filtering process for a light curve of a typical g' $=18.9$ WD in medium-deep field 3 . The total number of measurements and the number of dropouts $\left(\Delta F / \sigma_{\mathrm{lc}} \geqslant 5\right)$ are shown in the lower right of each panel. Dropout candidates are plotted as triangles. (a) Raw light curve before any filtering. Error bars are equivalent to the reported measurement uncertainties. Note the large number (1977) of dropout candidates. (b) Light curve after applying the chip location-based filters described in Section 2.4. (c) Light curve after removing measurements in which neighboring stars show large deviations from the median flux level or large scatter. (d) Light curve after de-correlating against the neighboring star relative flux and re-scaling the measurement uncertainties by adding the reported uncertainties in quadrature with the standard deviation of the light curve in each filter. This tends to inflate the error bars and pushes the vast majority of dropout events below the $5 \sigma$ cutoff. (e) Light curve after the final level of photometry-based filtering. In this stage, we compare the CCD pixel positions of the stars during dropout events with known masked regions of the CCD array. Two dropout events remain after all photometry-based filters. Postage stamp images are downloaded and visually inspected for the remaining dropout events.

(A color version of this figure is available in the online journal.) 


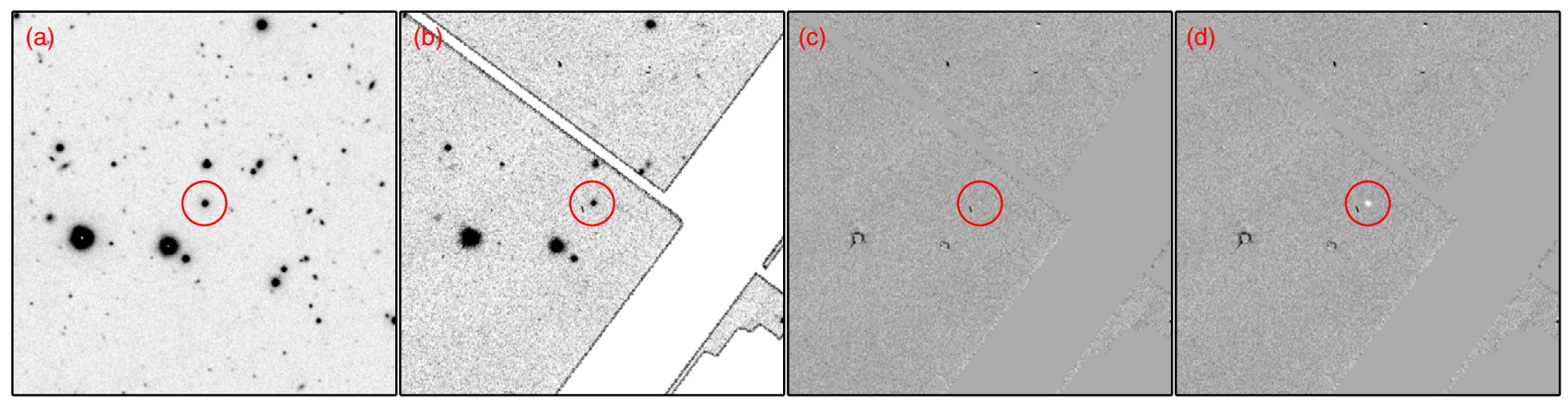

Figure 5. Candidate $z$-band eclipse with a reported depth of 53\% that was not filtered by the automated filtering techniques described in Section 2.4. (a) 5.8 hr stack of the 2.5 $\times 2.5$ region centered on the target. The target WD is circled. (b) The same field of view as panel (a) from the single exposure corresponding to the reported $53 \%$ deep eclipse. (c) A difference image of the stack in panel (a) convolved and scaled to match the PSF and subtracted from the dropout image in panel (b). Note that all stars—including the target—show no detectable residual flux. (d) Same as panel (c) with a synthetic 53\% eclipse injected onto the target before the image subtraction. The negative residuals on the target are clearly evident.

(A color version of this figure is available in the online journal.)

$$
\begin{aligned}
1-F(p, b) & = \begin{cases}0 & 1+p<b \\
\frac{1}{\pi}\left[p^{2} \kappa_{0}+\kappa_{1}-\sqrt{\frac{4 b^{2}-\left(1+b^{2}-p^{2}\right)^{2}}{4}}\right] & |1-p|<b \leqslant 1+p \\
p^{2} & b \leqslant 1-p \\
1 & b \leqslant p-1,\end{cases} \\
b(t) & \approx a \sqrt{\sin ^{2}\left(\Omega+\omega t+\alpha_{0}\right)+\sin ^{2} \theta \cos ^{2}\left(\Omega+\omega t+\alpha_{0}\right)}
\end{aligned}
$$

Equation (2) for $b(t)$ gives the sky-projected center-to-center distance between the star and planet as a function of time $(t)$. $\Omega$ is the longitude of the ascending node of the planet's orbit, $a$ is the semi-major axis of the orbit, $\omega$ is the angular frequency of the orbit, $\theta$ is the inclination of the planet's orbit, and $\alpha_{0}$ is the phase of inferior conjunction. Minimizing Equation (2) leads to the smallest sky-projected separation over the orbit, $b_{0}=R_{\mathrm{WD}} \cos \theta$.

In order to determine the likelihood that a particular $\Delta F$ could be caused by an eclipse of the WD, we calculate the probability of eclipses as a function of the eclipse depth. First, we make some assumptions for physical parameters that are mostly constant within the parameter region of interest. We assume that $M_{\mathrm{WD}}=0.6 M_{\odot}, R_{\mathrm{WD}}=0.012 R_{\odot}$, all theoretical companions are on circular orbits, there is no limb darkening, and $240 \mathrm{~s}$ is the integration time for every exposure. The probability of measuring an eclipse depth $\langle\Delta F(p, b)\rangle$ at time $t$ averaged over an exposure time of $\Delta t$ is

$$
\langle\Delta F(p, b)\rangle=\frac{1}{\Delta t} \int_{t_{0}}^{t 0+\Delta t} F(p, b) d t .
$$

Eclipses will only occur if $\left|b_{0}\right|<1+p$, and therefore the probability that a randomly oriented, circular orbit will eclipse is

$$
P_{\text {eclipse }}=\frac{R_{p}+R_{\mathrm{WD}}}{a} .
$$

Although systems with $\left|b_{0}\right|<1+p$ will eclipse at some time during the orbit, the fraction of orbital phase covered during the eclipse is small. The probability that any part of an eclipse will overlap with the integration time of our survey is

$$
P_{\text {phase }}=\frac{T_{\mathrm{dur}}+E}{P},
$$

where $P$ is the orbital period, $T_{\text {dur }}$ is the eclipse duration, and $E$ is the integration time.

For eclipses with durations shorter or equal to the exposure time, the likelihood of any given measurement being in eclipse is then the sum of the probabilities for all possible orbital configurations that would produce an observed eclipse of depth $m$. For example, a measurement with $m=0.1$ could be caused by a very small planet transiting slowly across the face of the star with a transit duration approximately equal to the exposure time. Alternatively, an eclipse of a much larger planet causing a complete occultation of the WD on a very short-period orbit would streak across the face of the star with a transit duration much shorter than the exposure time. The mean flux during the exposure may look identical in these two cases. Both of these cases and all other situations that could cause an observed eclipse depth $m$ must be given the appropriate weight in the final likelihood calculation. Figure 6 shows the eclipse depth probability distributions for a few hypothetical scenarios.

By the definition of our eclipse detection algorithm, each exposure is sensitive to eclipses of depth $m \geqslant 5 \Delta F / \sigma_{\mathrm{lc}}$. By integrating over all scenarios that would cause an observed eclipse depth greater than or equal to $5 \Delta F / \sigma_{\text {lc }}$ for every measurement, we derive the probability that we could have detected an eclipse during each exposure if $\eta=1$. The inverse of the summed probabilities over all exposures for all light curves gives a total number of expected eclipses for the survey as a Poisson expectation value for the rate of eclipses (Figure 7). We then compare this Poisson distribution for the expected number of eclipses with the lack of detected eclipses for many values of $a, p$, and $\eta$.

\subsection{Occurrence Constraints}

If we treat the number of expected eclipses as a Poisson expectation value $(\lambda)$, then the probability that we should detect 

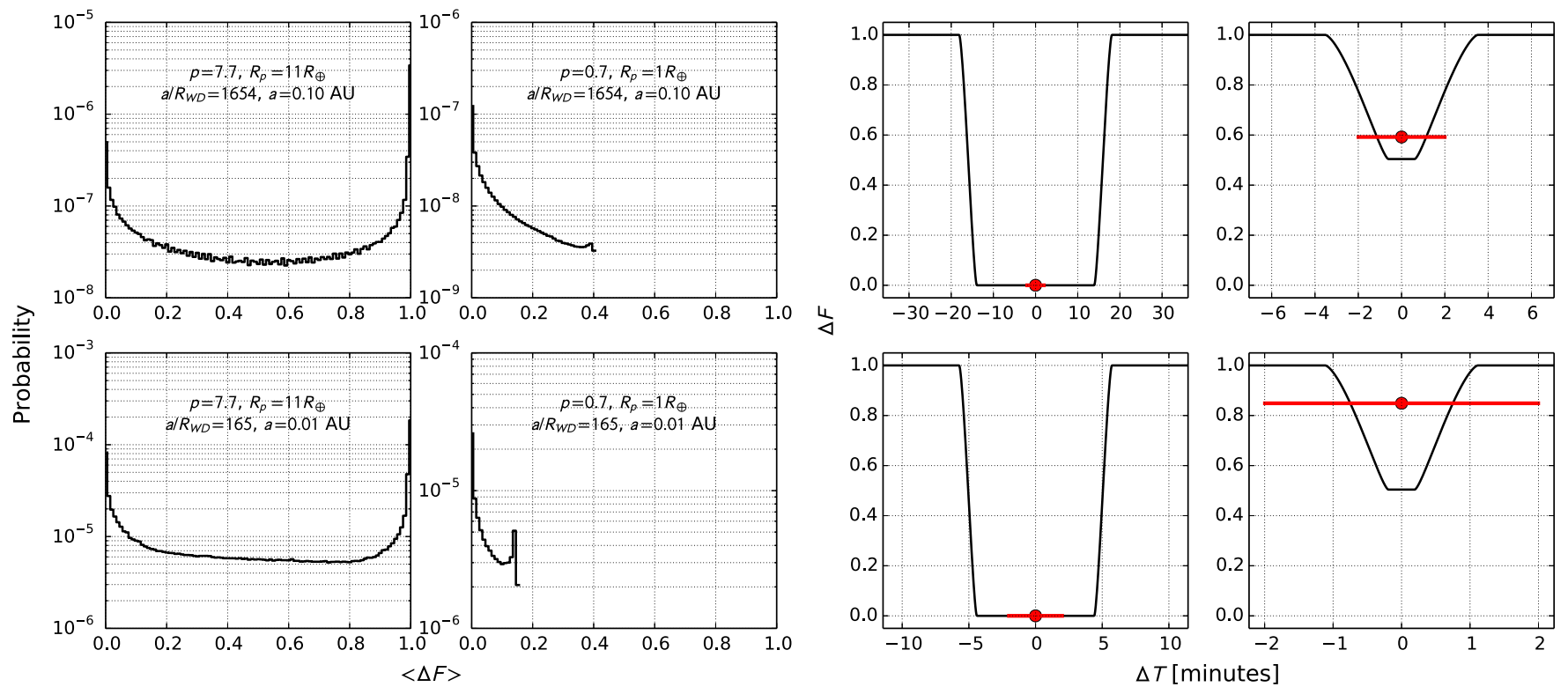

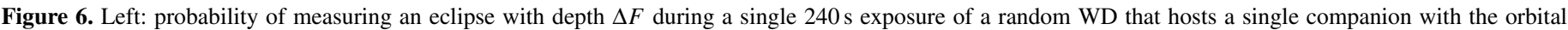

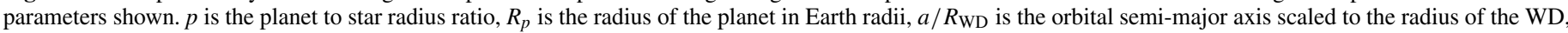

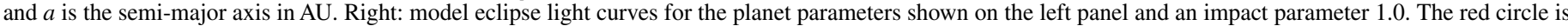

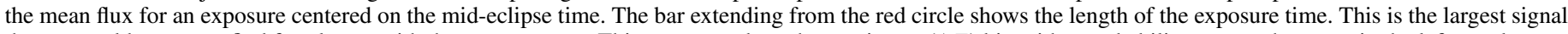
that we could expect to find for planets with these parameters. This corresponds to the maximum $\langle\Delta F\rangle$ bin with a probability greater than zero in the left panel.

(A color version of this figure is available in the online journal.)

$k$ eclipses is

$$
P(k, a, p)=\frac{\lambda(\eta, a, p)^{k} \exp (-\lambda(\eta, a, p))}{k !} .
$$

Since we have zero detected eclipses, this can be simplified to $P(0, a, p)=\exp (-\lambda(\eta, a, p))$. By setting $P(0, a, p)$ equal to a confidence interval $C$ and decomposing $\lambda(a, p)$ into the expectation value of eclipses if the planet occurrence rate is equal to $1\left(\lambda_{1}(a, p)\right)$ multiplied by the actual planet occurrence rate $(\eta)$, we derive the maximum planet occurrence rate that is compatible with the observations at a confidence level of $C$

$$
\eta \leqslant \frac{\ln (1-C)}{\lambda_{1}(a, p)}
$$

assuming that the planet occurrence rate is constant as a function of $a$ and $p$.

\section{DISCUSSION}

Although we find no convincing detections of eclipses with durations consistent with substellar objects, we are still able to place strong constraints on the WD-hosted planet occurrence rate. Figures 8 and 9 show the maximum occurrence rate that is consistent with our observations at $95 \%$ and $68 \%$ confidence levels assuming $R_{\mathrm{WD}}=0.012 R_{\odot}$ and $M_{\mathrm{WD}}=0.6 M_{\odot}$. This should be a relatively good approximation since the masses and radii of most WDs fall close to these values. For each reported occurrence rate $(\eta)$, we first state the value corresponding to the maximum allowable occurrence rate averaged over the specified region of interest for the $95 \%$ confidence limit, and then the $68 \%$ confidence limit immediately following in parenthesis. For example, our results suggest that less than $0.4 \%(0.2 \%)$ of WDs host planets with radii greater than $\sim 2$ Earth radii and semimajor axes between 0.002 and 0.01 AU. $0.4 \%$ is the maximum occurrence rate allowed by our data at $95 \%$ confidence and $0.2 \%$ is the same for a confidence level of $68 \%$.

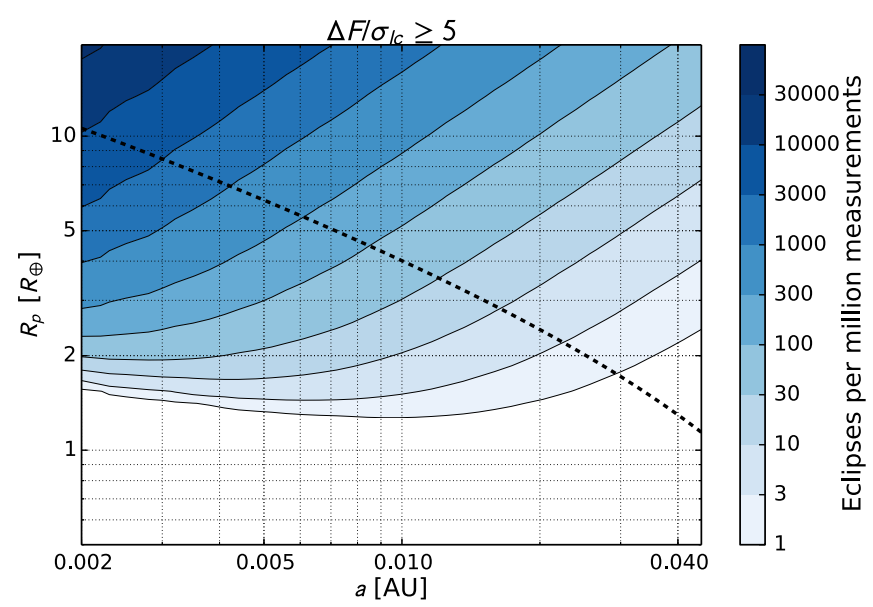

Figure 7. Expected detectable eclipse rate per million exposures of the mediumdeep survey. An eclipse is deemed detectable if the depth is greater or equal to five times the measurement uncertainty. The measurement uncertainty is calculated by adding the reported uncertainty in quadrature with the standard deviation of the light curve on a per-filter basis. The dashed line marks the point at which the eclipse duration is equal to the integration time. Eclipses caused by objects with parameters that fall in the region above and to the right of the dashed line will have eclipses that may span multiple adjacent exposures. Our assumption that each light curve measurement is independent is invalid in this regime and our expected eclipse rate will be slightly overestimated.

(A color version of this figure is available in the online journal.)

It is an interesting exercise to break up the two-dimensional occurrence limits into regions that correspond to classes of planets that we are more familiar with orbiting main-sequence stars. Other studies have shown similarities between the architectures of exoplanetary systems around low-mass M-dwarfs with the moons of Jupiter (Muirhead et al. 2012) and scaled-down versions of our solar system or exoplanetary systems around more massive stars. If we scale down the orbital distances of the known 

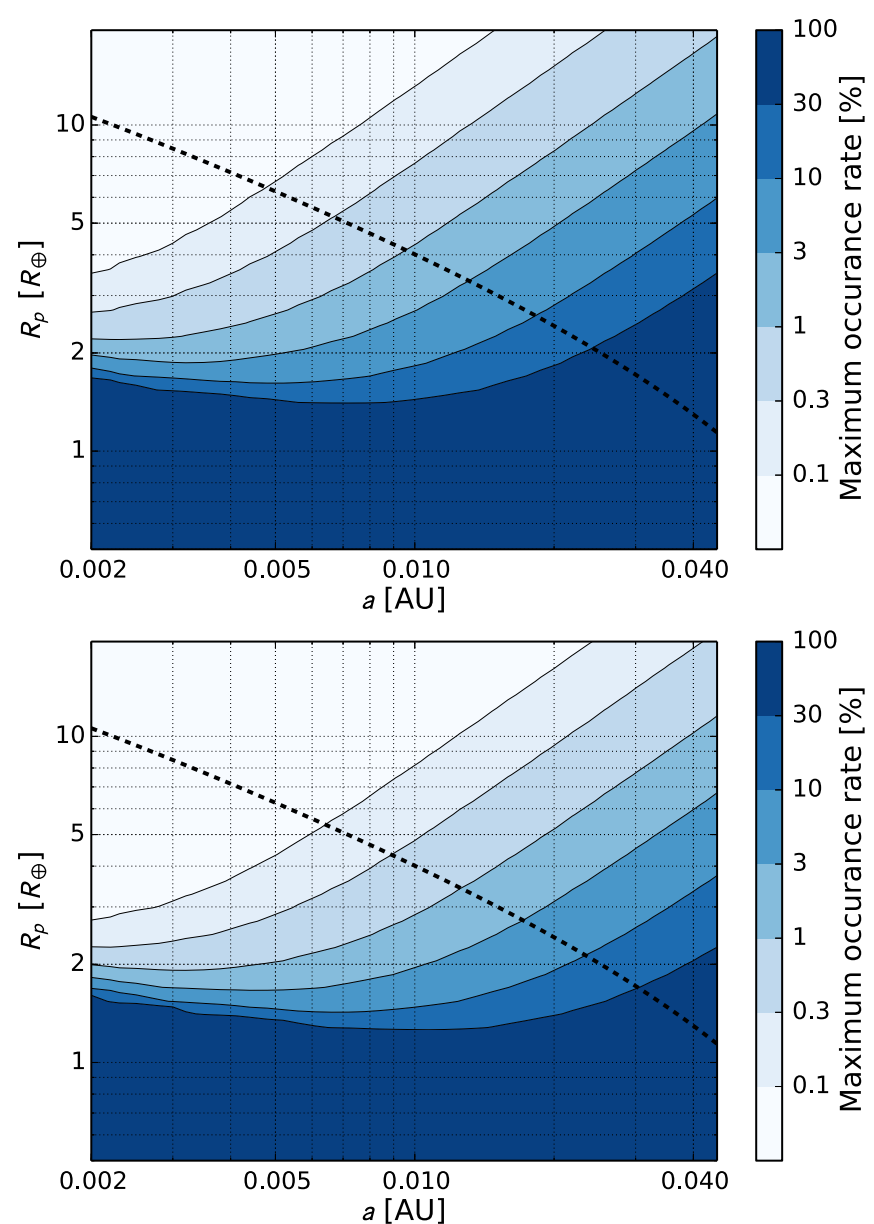

Figure 8. Top: maximum planet occurrence rate compatible with the observations at $95 \%$ confidence. Bottom: maximum planet occurrence rate compatible with the observations at $68 \%$ confidence. In both panels, the dashed line is the same as in Figure 7. The maximum occurrence rates will be slightly underestimated in the region to the upper right of this dashed line.

(A color version of this figure is available in the online journal.)

exoplanet population, then we can look at the occurrence limits in a few interesting regimes; hot Jupiters, hot super-Earths, and HZ super-Earths.

The Roche limit for a fluid body with mean density $\rho_{p}$ orbiting a WD with density $\rho_{\mathrm{WD}}$ and radius $R_{\mathrm{WD}}$ can be approximated as

$$
L_{R} \approx 2.44 R_{\mathrm{WD}}\left(\frac{\rho_{\mathrm{WD}}}{\rho_{p}}\right)^{1 / 3}
$$

For our assumed WD properties, the Roche limit for a Jupiterlike planet is $L_{R} \approx 0.01 \mathrm{AU}$. It is not surprising that we do not detect any Jupiter-sized objects inside 0.01 AU. However, we can equate a population of Jupiter-sized planets orbiting between 0.01 and $0.04 \mathrm{AU}$ to the hot Jupiters observed orbiting very close to solar-type stars. In this regime, an eclipse duration is slightly longer than the duration of a single exposure. Therefore, our expectation value for eclipses is slightly overestimated, however, we do not expect this to be the dominant source of error in the occurrence rate limits. The mean maximum occurrence rate for WD-hosted hot Jupiters $\left(R=10-20 R_{\oplus}\right)$ is $0.5 \%(0.2 \%)$. Indicating that hot Jupiters around WDs are very rare or non-existent. This is in good agreement with the frequency of hot Jupiters around solar-type stars, measured to

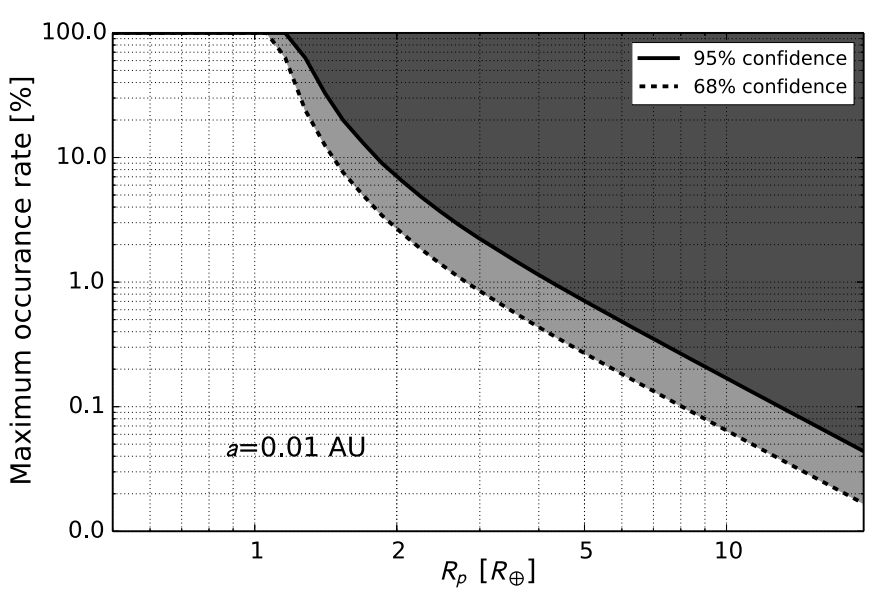

Figure 9. Maximum planet occurrence rate consistent with our data as a function of planet radius at a semi-major axis of $a=0.01 \mathrm{AU}$ for confidence levels of $95 \%$ (solid) and $68 \%$ (dashed). Shaded regions are disfavored by our data. This plot represents a slice through Figure 8 at $a=0.01 \mathrm{AU}$.

be between $0.3 \%$ and $1.5 \%$ (Marcy et al. 2005; Gould et al. 2006; Cumming et al. 2008; Howard et al. 2011; Mayor et al. 2011; Wright et al. 2012).

A rigid body can orbit slightly closer to the WD without being tidely disrupted. Planets with radii larger than $\sim 1.5 R_{\oplus}$ generally have densities lower than that of the Earth and likely have an extended gas-dominated atmosphere (Weiss \& Marcy 2014). However, some super-Earths with slightly larger radii have high densities consistent with a rocky composition, e.g., CoRoT-7b (Léger et al. 2009), Kepler-20b (Gautier et al. 2012), and Kepler-19b (Ballard et al. 2011). This class of planets may be the remaining cores of evaporated gas giant planets (Hébrard et al. 2004). Our results suggest that less than $1.5 \%(0.6 \%)$ of WD host planets with radii between 2.0 and $5.0 R_{\oplus}$ orbiting with semi-major axis between 0.005 and $0.01 \mathrm{AU}$. Howard et al. (2012) measure an occurrence rate of $13 \%$ for $2-4 R_{\oplus}$ planets with orbital periods shorter than 50 days. However, the occurrence rate drops with shorter orbital periods to $2.5 \%$ for periods shorter than 10 days. Our lack of detections indicate that hot super-Earths are almost certainly less common around WDs than they are around solar-type stars.

Perhaps the most interesting planets to consider are those that have an equilibrium temperature such that they could sustain liquid water on their surfaces. Since WDs cool and decrease in luminosity as they age, the HZ boundaries also change as a function of time. Agol (2011) defines the WD continuous habitable zone (CHZ) as the range of semi-major axis that would be within the $\mathrm{HZ}$ for a minimum of $3 \mathrm{Gyr}$ and also outside of the tidal destruction radius for an Earth-density planet. For a $0.6 M_{\odot}$ $\mathrm{WD}$, this corresponds to a semi-major axis between 0.005 and 0.02 AU. Our data show that planets in the $\mathrm{CHZ}$ with radii between 2 and $5 R_{\oplus}$ could be present around no more than $3.4 \%$ $(1.3 \%)$ of WDs. This is significantly less than the predicted frequency of Earth-size planets in the HZ of solar-type stars ( 22\%; Petigura et al. 2013).

A large population of short-period planets orbiting solar-type and M-dwarf stars has been observed. We might expect WDs to host similar planets if they can reform from a post-giant phase debris disk or migrate from larger orbital distances once the star becomes a WD. However, our observations are quite sensitive to planets larger than the Earth orbiting close to the WD, and the lack of any eclipses suggests that these processes are highly 

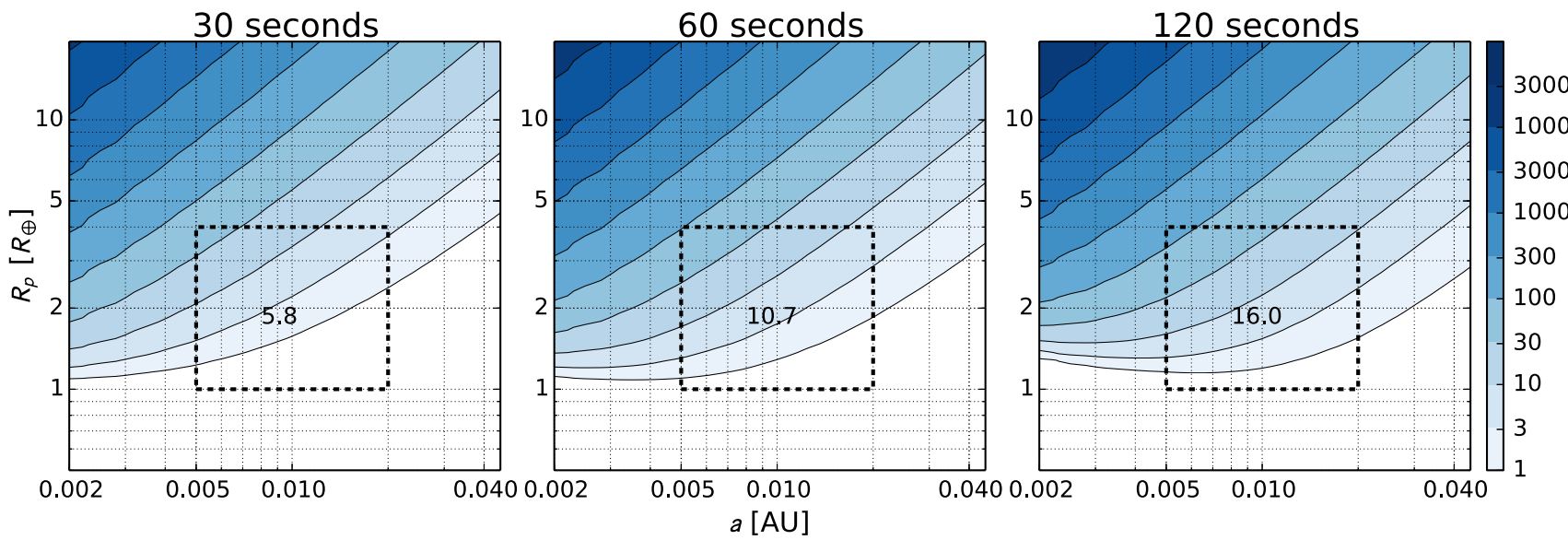

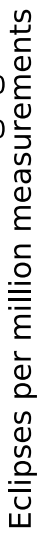

Figure 10. Expected detectable eclipse rates calculated as described in Section 3.1 for hypothetical surveys using the Pan-STARRS1like throughput with different exposure times. The numbers within the dashed box indicate the mean eclipse rate in that region of parameter space. Shorter exposure times give increased eclipse detectability for the shortest-period objects within $\sim 0.03 \mathrm{AU}$, but planets orbiting this close to their host WD would likely be ripped apart by tidal forces. Although the mean eclipse rate in the region of interest goes up with longer exposure times, this is reversed if you consider a fixed total survey exposure time (take twice as many $60 \mathrm{~s}$ exposures as $120 \mathrm{~s}$ exposures, etc.). However, the eclipse rates remain nearly constant, indicating that the best way to increase sensitivity in this regime is to increase the number of epochs observed (larger number of WDs and/or higher cadence).

(A color version of this figure is available in the online journal.)

inefficient if they occur at all. There are very few planets in short-period orbits around WDs.

\subsection{Future Survey Design}

Since eclipse times are generally shorter than the four minute exposure times for the medium-deep survey, we explore the idea of designing a similar survey with shorter exposure times and decreased sensitivity to shallow eclipses. This would cause less dilution of the eclipse signals over the duration of the exposure. We recalculate the expected eclipse rates for exposure times of 30,60 , and $120 \mathrm{~s}$ scaling the measured noise properties from our $240 \mathrm{~s}$ data. We use the mean eclipse rate for planets with radii between 1 and $5 R_{\oplus}$ orbiting between 0.005 and $0.02 \mathrm{AU}$ as a metric for comparison. Figure 10 illustrates the result. We find that decreasing the exposure time gives a modest boost in sensitivity to these planets for a given total survey exposure time. The most dramatic increase in sensitivity when going to short exposure times is for the very short-period planets orbiting interior to $0.003 \mathrm{AU}$. However, planets are not able to withstand the tidal forces this close to the WD so we would not expect planets to exist in this regime. The expected eclipse rate in our region of interest is dominated by the signal to noise of the individual detections. Although the eclipses are diluted by long exposure times, this is balanced by the increased gain in sensitivity to these shallow, diluted eclipses due to the greater signal to noise obtained in longer exposures. This suggests that the best way to detect these Earth-to-Neptune-size planets in the WD CHZ may be to increase the etendue of the survey to detect more WDs on a greater number of epochs by covering a large area of the sky at high cadence. The ATLAS (Tonry 2011) and Large Synoptic Survey Telescope (Ivezic et al. 2008) surveys should be ideal for detecting these extremely rare events.

\subsection{Pan-STARRS1 $3 \pi$}

The Pan-STARRS $13 \pi$ survey covers $30,000 \mathrm{deg}^{2}$ with approximately 60 observational epochs per object (Kaiser et al. 2010; Magnier et al. 2013). The depth and cadence are inferior to that of the medium deep fields, but the huge amount of sky observed makes it interesting to explore the contribution that this survey could make to the occurrence rate limits if we were to perform a similar analysis on a combined data set.

We start with an order of magnitude estimate of the number of WDs we would expect to find in the $3 \pi$ survey data via reduced proper motion. The exposure times for the $3 \pi$ survey are $60 \mathrm{~s}$ versus $240 \mathrm{~s}$ for the medium deep fields, but let us assume that our ability to detect WDs is limited by the length of the observational baseline and not by signal to noise of the detections. Since the sky coverage is a factor of $\sim 400$ greater in the $3 \pi$ survey it is reasonable to scale the number of astrometrically selected WDs found in the medium-deep fields (661) by 400 . Therefore, we expect to find $\sim 30,000$ WDs via reduced proper motion in the $3 \pi$ data. Since each WD is observed 60 times this gives a total of 1.8 million measurements. The shorter exposure times increase our sensitivity to very short duration eclipses, however, the largest gain in sensitivity is to planets orbiting well inside the tidal destruction radius (see Figure 10 and Section 4.1). Combining these 1.8 million epochs with the 4.3 million epochs from the medium-deep fields increases our total number of measurements by a factor of 1.4 and strengthens (decreases) our maximum occurrence constraints by this same factor. This $\sim \sqrt{2}$ improvement would not change our primary conclusion that planets around WDs are rare.

\section{CONCLUSIONS}

Our systematic search for eclipses of WDs in the Pan-STARRS1 medium-deep fields places strong constraints on the WD planet occurrence rates. We analyze a sample of $\sim 3000$ WDs selected via proper motion and color along with a control sample of $\sim 1200$ stars. These WDs were observed for five years on over 4.3 million epochs.

We search for potential eclipses by identifying low outliers in the light curves. A total of 133 candidate eclipses are identified after applying a series of photometry then image-based filters to remove outliers caused by weather, CCD artifacts, or an improperly modeled PSF. After a visual inspection of all candidates, we find none that are consistent with an eclipse or occultation by a substellar object.

We calculate the number of expected eclipses if every WD hosted at least one planet $(\eta=1)$ by convolving a trapezoidal 
transit model with the survey exposure time and integrating over all possible geometric orientations and many values of $R_{p}$ and $a$. The expected number of eclipses is treated as a Poisson expectation value for the rate of events that are converted into $95 \%(68 \%)$ confidence intervals. We then invert these rates to obtain the maximum value of $\eta$ consistent with our data.

Our results suggest that hot Jupiters around WDs are at least as rare as they are around solar-type stars, occurring around no more than $0.5 \%(0.2 \%)$ of WDs. Hot super-Earths occur around no more than $1.5 \%(0.6 \%)$ of stars, and super-Earths in the $\mathrm{CHZ}$ are present around no more than $3.4 \%(1.3 \%)$ of WDs. All evidence presented in this study indicate that shortperiod planets around WDs are significantly less abundant than short-period planets orbiting main-sequence stars.

The Pan-STARRS1 Surveys (PS1) have been made possible through the contributions of the Institute for Astronomy, the University of Hawaii, the Pan-STARRS Project Office, the Max-Planck Society and its participating institutes, the Max Planck Institute for Astronomy, Heidelberg and the Max Planck Institute for Extraterrestrial Physics, Garching, The Johns Hopkins University, Durham University, the University of Edinburgh, Queen's University Belfast, the Harvard-Smithsonian Center for Astrophysics, the Las Cumbres Observatory Global Telescope Network Incorporated, the National Central University of Taiwan, the Space Telescope Science Institute, the National Aeronautics and Space Administration under grant No. NNX08AR22G issued through the Planetary Science Division of the NASA Science Mission Directorate, the National Science Foundation under grant No. AST-1238877, the University of Maryland, and Eotvos Lorand University (ELTE). Support for this work was provided by National Science Foundation grant AST-1009749. Finally, we thank Professor Andrew Gould for his critical review and extremely helpful suggestions that greatly enhanced the quality of this work.

\section{REFERENCES}

Agol, E. 2011, ApJL, 731, L31

Alard, C. 2000, A\&AS, 144, 363

Bakos, G., Noyes, R. W., Kovács, G., et al. 2004, PASP, 116, 266

Ballard, S., Fabrycky, D., Fressin, F., et al. 2011, ApJ, 743, 200

Borucki, W. J., Koch, D., Basri, G., et al. 2010, Sci, 327, 977

Burleigh, M. R., Hogan, E., Dobbie, P. D., Napiwotzki, R., \& Maxted, P. F. L. 2006, MNRAS, 373, L55
Cumming, A., Butler, R. P., Marcy, G. W., et al. 2008, PASP, 120, 531

Debes, J. H., Hoard, D. W., Kilic, M., et al. 2011, ApJ, 729, 4

Debes, J. H., Sigurdsson, S., \& Woodgate, B. E. 2005, AJ, 130, 1221

Devor, J. 2005, ApJ, 628, 411

Drake, A. J., et al. 2010, e-print (arXiv:1009.3048)

Faedi, F., West, R. G., Burleigh, M. R., Goad, M. R., \& Hebb, L. 2011, MNRAS, 410, 899

Farihi, J., Becklin, E. E., \& Zuckerman, B. 2008, ApJ, 681, 1470

Farihi, J., Zuckerman, B., \& Becklin, E. E. 2005, AJ, 130, 2237

Gaudi, B. S. 2012, ARA\&A, 50, 411

Gautier, T. N., III, Charbonneau, D., Rowe, J. F., et al. 2012, ApJ, 749, 15

Gould, A., Udalski, A., An, D., et al. 2006, ApJL, 644, L37

Hartman, J. D., Gaudi, B. S., Holman, M. J., et al. 2008, ApJ, 675, 1233

Hébrard, G., Lecavelier Des Étangs, A., Vidal-Madjar, A., Désert, J.-M., \& Ferlet, R. 2004, in ASP Conf. Ser. 321, Extrasolar Planets: Today and Tomorrow, ed. J. Beaulieu, A. Lecavelier Des Etangs, \& C. Terquem (San Francisco, CA: ASP), 203

Hogan, E., Burleigh, M. R., \& Clarke, F. J. 2009, MNRAS, 396, 2074

Howard, A. W., Johnson, J. A., Marcy, G. W., et al. 2010a, ApJ, 721, 1467

Howard, A. W., Johnson, J. A., Marcy, G. W., et al. 2011, ApJ, 726, 73

Howard, A. W., Marcy, G. W., Bryson, S. T., et al. 2012, ApJS, 201, 15

Howard, A. W., Marcy, G. W., Johnson, J. A., et al. 2010b, Sci, 330, 653

Ivezic, Z., et al. 2008, e-print (arXiv:0805.2366)

Johnson, J. A., Fischer, D. A., Marcy, G. W., et al. 2007, ApJ, 665, 785

Jura, M., Farihi, J., \& Zuckerman, B. 2009, AJ, 137, 3191

Kaiser, N., Burgett, W., Chambers, K., et al. 2010, Proc. SPIE, 7733, 77330E

Kilic, M., Gould, A., \& Koester, D. 2009, ApJ, 705, 1219

Kovács, G., Zucker, S., \& Mazeh, T. 2002, A\&A, 391, 369

Léger, A., Rouan, D., Schneider, J., et al. 2009, A\&A, 506, 287

Magnier, E. A., Schlafly, E., Finkbeiner, D., et al. 2013, ApJS, 205, 20

Mandel, K., \& Agol, E. 2002, ApJL, 580, L171

Marcy, G., Butler, R. P., Fischer, D., et al. 2005, PThPS, 158, 24

Mayor, M., Marmier, M., Lovis, C., et al. 2011, arXiv:1109.2497

Muirhead, P. S., Johnson, J. A., Apps, K., et al. 2012, ApJ, 747, 144

Mullally, F. R. 2007, PhD thesis, The Univ. Texas at Austin

Mustill, A. J., Veras, D., \& Villaver, E. 2014, MNRAS, 437, 1404

Nutzman, P., \& Charbonneau, D. 2008, PASP, 120, 317

Petigura, E. A., Howard, A. W., \& Marcy, G. W. 2013, PNAS, 110, 19273

Pollacco, D. L., Skillen, I., Collier Cameron, A., et al. 2006, PASP, 118,1407

Robin, A. C., Reylé, C., Derrière, S., \& Picaud, S. 2003, A\&A, 409, 523

Schlafly, E. F., Finkbeiner, D. P., Jurić, M., et al. 2012, ApJ, 756, 158

Schwarzenberg-Czerny, A. 1989, MNRAS, 241, 153

Steele, P. R., Burleigh, M. R., Farihi, J., et al. 2009, A\&A, 500, 1207

Tonry, J. L. 2011, PASP, 123, 58

Tonry, J. L., Stubbs, C. W., Kilic, M., et al. 2012, ApJ, 745, 42

Weiss, L. M., \& Marcy, G. W. 2014, ApJL, 783, L6

Wright, J. T., Marcy, G. W., Howard, A. W., et al. 2012, ApJ, 753, 160

Xu, S., Jura, M., Koester, D., Klein, B., \& Zuckerman, B. 2014, ApJ, 783,79

Zuckerman, B., \& Becklin, E. E. 1992, ApJ, 386, 260

Zuckerman, B., Melis, C., Klein, B., Koester, D., \& Jura, M. 2010, ApJ, 722,725 